\section{MRI estimates size of normal kidneys more accurately than standard methods}

Estimates of kidney length and volume are surrogates for renal functional reserve. These estimates have typically been generated using two-dimensional ultrasonography and an ellipsoid formula that assumes a certain kidney shape. On the basis of a retrospective analysis, Cheong et al. have concluded that the ellipsoid equation underestimates kidney length and volume, and that three-dimensional MRI techniques more accurately determine these renal parameters.

Compared with a water displacement test, the ellipsoid formula (using lengths obtained by MRI) underestimated the volume of five freshly excised porcine kidneys by $21-29 \%$. By contrast, an MRI-based disk-summation method underestimated volumes by only 0 $5 \%$. In 150 retrospectively identified patients (300 kidneys) who had undergone abdominal $\mathrm{MRI}$ scans for reasons other than renal disease, kidney volumes calculated using the ellipsoid formula were significantly smaller than disk-summation estimates $(P<0.0001)$; this was the case despite the fact that MRIbased, as opposed to ultrasonography-based, measurements of length, width and depth were used in all ellipsoid calculations.

MRI-based disk summation was shown to be an accurate, noninvasive method of estimating renal size, even at relatively modest spatial resolutions $\left(28 \mathrm{~mm}^{3}\right)$. The authors conclude that the estimates of normal kidney size that they obtained via MRI disk summation (mean volumes of $202 \mathrm{ml}$ and $154 \mathrm{ml}$ for men and women, respectively) are larger, and closer to true kidney size, than the current standard ranges (110-190 $\mathrm{ml}$ for men and $90-150 \mathrm{ml}$ for women), which are based on ultrasonography and the ellipsoid formula.

Original article Cheong B et al. (2007) Normal values for renal length and volume as measured by magnetic resonance imaging. Clin J Am Soc Nephrol 2: 38-45

\section{Is serum NGAL an accurate marker of renal function in pediatric CKD?}

A group in the US previously showed that serum levels of neutrophil gelatinase-associated lipocalin (NGAL) are markedly increased in children at risk of developing acute kidney injury following cardiac surgery. By examining NGAL's relationships with measured glomerular filtration rate (GFR) and with known predictors of GFR, these researchers have now investigated whether levels of this protein can be used to determine the severity of chronic kidney disease (CKD).

The trial included 45 children and adolescents (aged 6-21 years) with CKD stages 2-4. At enrollment, the following parameters were recorded: GFR measured as ioversol clearance, estimated GFR (eGFR) calculated using the Schwartz formula, and serum levels of creatinine, NGAL and cystatin C.

Serum NGAL and cystatin C levels were highly correlated (coefficient of correlation $r=0.74$ ), and both of these variables correlated with serum creatinine level, eGFR and measured GFR ( $r \geq 0.50$ for all). In patients with measured GFR of at least $30 \mathrm{ml} / \mathrm{min} / 1.73 \mathrm{~m}^{2}$, serum cystatin $C$, serum NGAL, serum creatinine and eGFR all showed good correlation with measured GFR $(r=0.45,0.52,0.70$ and 0.72 , respectively). In advanced CKD (measured GFR $<30 \mathrm{ml} / \mathrm{min} / 1.73 \mathrm{~m}^{2}$ ), serum NGAL was the best predictor of measured GFR $(r=0.62)$, followed by serum cystatin C $(r=0.41)$; neither serum creatinine level nor eGFR were good predictors of measured GFR in advanced CKD.

The authors contend that a panel of protein biomarkers (including serum NGAL) will become the preferred tool for predicting and monitoring progression of CKD.

\section{Original article Mitsnefes MM et al. (2007) Serum neutrophil gelatinase-associated lipocalin as a marker of renal function in children with chronic kidney disease. Pediatr Nephrol 22: 101-108 \\ Tolvaptan increases serum sodium concentrations in hyponatremic patients}

Hyponatremia is a predictor of death and morbidity in patients with heart disease, cirrhosis or neurologic disorders. Two recent identically designed, randomized, placebo-controlled, multicenter studies investigated the effects of tolvaptan, an oral vasopressin $\mathrm{V}_{2}$-receptor antagonist, on hyponatremia.

SALT-1 and SALT-2 enrolled a total of 448 outpatients with chronic heart failure, cirrhosis, 